\title{
Kinematics model for the wheeled vehicle tractive performance determination on Sepang's peat terrain in Malaysia
}

\begin{abstract}
Mobility of the LGP-30 wheeled vehicle is simulated by using the kinematics model developed in this paper based on the vehicle's tyre-terrain interaction model. The kinematics model on slippage represents that slippage is a major function of vehicle sinkage. The simulation results show that the LGP-30 wheeled vehicle would not be able to traverse on Sepang's peat terrain as the vehicle sinkage is significantly higher than surface mat thickness and vehicle ground clearance as well.
\end{abstract}

Keyword: Peat terrain; Kinematics model; LGP-30 wheeled vehicle; Mobility 